\section{INTERMAX: A program for orthogonal analytical rotation by the intermax criterion}

\section{JOSEPH L. BALLOUN and A. BEN OUMLIL University of Dayton, Dayton, Ohio}

In this paper, we describe a FORTRAN IV program, INTERMAX, that performs the intermax rotation of orthogonal factor structure matrices. Furthermore, the program enables new statistical tests for the number of factors, the presence of simple structure, the communality of a variable, the eigenvalue of a rotated factor, and the significance of a rotated loading.

\section{THE NEED FOR THE INTERMAX ROTATION METHOD}

A continuing challenge in factor analysis and in related techniques is the "seeming difficulty of applying tests of significance to the results of factor analyses" (Cattell, 1966, p. 332). For example, there is continuing ambiguity about how to choose the number of factors in exploratory factor analysis. There are many significance tests available in factor analysis (e.g., Cattell, 1978; Harman, 1967; Kim \& Mueller, 1978). According to Kim and Mueller (1978), the maximum likelihood tests often yield too many factors. Researchers often choose the number of factors on the basis of judgments of substantive importance of the factors, optimal simple structure in the rotated solution, interpretability or invariance, or a combination of criteria rather than maximum likelihood or other significance tests alone (Kim \& Mueller, 1978).

Similarly, rules of thumb are often used for the significance of a rotated factor loading. Different sources suggest 0.30 to 0.59 as the cutoff for a significant rotated loading (e.g., Hair, Anderson, Tatham, \& Grablowsky, 1979; W. Meredith [personal communication, spring, 1967]; Oumlil, 1983; L. Wolins [personal communication, fall, 1965]).

The available methods are not very general. For example, the maximum likelihood methods usually are concerned with the unrotated solution from a matrix of Pearsonian correlations. Multivariate normality is usually assumed for hypothesis-testing purposes. Different statistical methods are appropriate for the results of different

We are indebted to two anonymous reviewers and the editor of this journal who provided many valuable suggestions and constructive criticisms of earlier drafts of this article.

Dara C. Merenski developed extensive program documentation and assisted in debugging the Fortran IV program which supports the computations needed to implement the methods described in this article.

J. L. Balloun is with the Department of Management and A. Ben Oumlil is with the Department of Marketing, both at the University of Dayton, Dayton, OH 45469. types of dimensional analyses (e.g., nonmetric multidimensional scaling of proximities vs. maximum likelihood factor analysis). It is difficult to statistically compare the results of different types of analyses of the same data by different algorithms. For these reasons, it is important to seek a more general solution to the problem of hypothesis testing in rotated factor analysis solutions, or dimensional analyses in general.

\section{STATEMENT OF PURPOSE}

The purposes of this article are (1) to explain the rationale for a new method of hypothesis testing in rotated dimensional analyses, (2) to explain the computational methods used in the program, and (3) to illustrate the method with a small data set. (More detailed instructions on how to use the program are provided in the user's guide to the INTERMAX program.)

\section{RATIONALE FOR THE INTERMAX ROTATION METHOD}

\section{Theoretical Basis}

A long-standing issue in orthogonal factor analysis is how to objectively arrive at a simple-structure rotation of the factor structure matrix (Kim \& Mueller, 1978; Thurstone, 1947). The rationale for the intermax method starts from considering the factor structure matrix as a two-way factorial analysis of variance. The squared factor loadings are the dependent variable scores observed in each cell of the design. Different variables constitute levels of one independent variable, and the different factors constitute levels of the other independent variable. The intermax rotation maximizes the relative amount of squared factor loading variation that is due to the interaction of factors and variables (Balloun \& Kearns, 1975). The rationale for the intermax rotation is simpler than that of the varimax rotation, and it is related both to other rotation methods and to the analysis of variance (Balloun \& Kearns, 1975).

\section{Computational Methods}

The intermax criterion is the ratio

$$
\text { INT }=\text { SSINT/SSTOT, }
$$

where INT is the criterion to be maximized by the rotation, SSINT is the sum of squares interaction for squared factor loadings, and SSTOT is the sum of squares total for squared factor loadings.

Balloun and Kearns (1975) concluded that the intermax solution was often similar to the normalized varimax solution, and that this similarity tended to increase as the number of factors increased. This observation led to the following computational procedure. First the varimax ro- 
tation is calculated. Then the intermax rotation for each pair of factors is found by Newton's first-order iteration method. The solution is repeated for all pairs of factors and for the entire set of all possible pairs of factors until the solution converges. (For specific mathematical details, see Balloun \& Kearns, 1975.)

\section{THE STATISTICAL TESTS}

\section{Test for the Number of Factors}

Determination of the number of factors is the first decision made in exploratory factor analysis. The Fortran IV program described in this paper limits the number of factors extracted to the maxima suggested by Kim and Mueller (1978, pp. 49-51), depending on the number of variables in the problem. Due to space restrictions, a maximum of $\mathbf{3 0}$ factors may be extracted. The intermax rotation method can be augmented to provide a significance test for the number of factors based on maximization of simple structure.

\section{Analysis of Variance of \\ Squared Factor Loadings}

The intermax rotation method considers the variables and the factors as the two independent variables and the squared factor loadings as the dependent variable. Suppose that significance tests could be developed for the analysis of variance effects in the matrix of squared factor loadings. Then a significant difference among variables would indicate communality differences in the population. Significant differences among factors after rotation are usually not of substantive interest, but they are useful in describing population differences in rotated factor magnitudes. A significant interaction effect would indicate simple structure in the population. If samples of variables were randomly chosen from specified populations of variables, then factorial invariance could be tested by the interaction effect.

\section{The Significance of Individual Factor Loadings}

If the sampling variance of each individual unsquared, rotated factor loading could be estimated, then it would also be possible to test the significance of each rotated loading. The intermax rotation is designed to achieve positive manifold and simple structure. Therefore, the appropriate null hypothesis would be for zero or negative loading.

In summary, the intermax rotation method suggests novel statistical tests of various hypotheses for the rotated solution. These hypothesis tests are useful alternatives and are complementary to those presently available (e.g., the maximum likelihood test for the number of factors).

\section{Estimation of Parameters and Sampling Variances}

By itself, the intermax rotation method does not yield estimates of sampling variances. Hypothesis-testing uses of the intermax rotation method assume that there are reasonable estimates of the sampling errors of the squared or original rotated factor loadings.

Two methods for estimating parameters and sampling variances are used in the program. If raw data are input, then the jackknife method is used to estimate both parameters and sampling variances. If a factor loading matrix or a correlation matrix is input, then a parametric method is used.

\section{JACKKNIFE PARAMETER AND SAMPLING VARIANCE ESTIMATES}

Lack of convenient estimates of sampling variance is a common problem in statistical analysis (Tukey, 1980). Even when variability or significance or confidence interval estimates are available, it is often assumed that the sample data are well conditioned (see Nie, Hull, Jenkins, Steinbrenner, \& Bent, 1975). For example, inferential tests in factor analysis typically assume that the data contain no outliers, are drawn from a single population, are homoscedastic, and are multivariately normal, and that the associated correlation matrix is "non-Heywood" (Harman, 1967). There is often doubt that these assumptions are sufficiently well met to allow adequate modeling of the data or statistical inferences by the usual procedures.

Many variables are far from normally distributed. For example, response latency, dichotomous variables, error scores, deviations from a social norm, and accident rates are usually not normally distributed. Normalizing transformations do not remedy all such problems. Correlation matrices are often not well conditioned. For example, missing data in survey research often create ill-conditioned correlation matrices. Variables such as income, amount of rainfall, the weight of hogs, and response frequency often exhibit heteroscedasticity when correlated with other variables.

In cases in which there is no efficient way to calculate sampling variances or the available methods make implausible assumptions, there are general methods for calculating sampling variance, confidence intervals, or significance levels. The jackknife method is the best such general method (Tukey, 1980), and it has been previously applied to estimating sampling errors with rotated factor loadings (Clarkson, 1979). It removes bias proportional to the reciprocal of sample size $(N)$, tends to reduce estimate bias, provides a means of estimating error variance and significance or confidence intervals when alternatives are unavailable or their assumptions are violated. The method is generally robust, but caution or data transformations may be necessary in special circumstances. (For further information on the rationale for the jackknife technique, see Balloun \& Oumlil, 1986; Bissell, 1977; Clarkson, 1979; Miller, 1974; and Mosteller \& Tukey, 1968.)

\section{Jackknife Computational Method}

The computational method involves the following steps (Balloun \& Oumlil, 1986; Bissell, 1977; Clarkson, 1979; 
Table 1

Definition of Symbols used in Jackknife Calculations

\begin{tabular}{cl}
\hline Symbol & \multicolumn{1}{c}{ Meaning } \\
\hline$\hat{\theta}$ & $\begin{array}{l}\text { A sample estimate of a population parameter calculated from } \\
\text { the total sample. }\end{array}$ \\
$\hat{\theta}_{-j}$ & $\begin{array}{l}\text { The sample estimate when the jth subgroup is omitted and } \\
\text { the estimate is found from the remaining data. }\end{array}$ \\
$\hat{\theta}_{* j}$ & $\begin{array}{l}\text { A jackknifed "pseudovalue" for each subgroup, computed } \\
\text { as in Equation } 2 .\end{array}$ \\
$\hat{\theta}_{*}$ & The mean of the $\hat{\theta}_{* j}$ values. \\
$N$ & The sample size. \\
$\phi$ & $\begin{array}{l}\text { The } \theta \text { estimate transformed to reduce possible nonnormality } \\
\text { or to avoid "out of range" estimates. The symbol may ap- } \\
\text { pear with various subscripts, as for } \theta .\end{array}$ \\
$r$ & $\begin{array}{l}\text { Number of subgroups used. } \\
s\end{array}$ \\
$k$ & Size of each subgroup. \\
& Number of parameters to be estimated.
\end{tabular}

Tukey, 1980; symbols are defined in Table 1): (1) Calculate the parameter estimate, $\hat{\theta}$, based on all sample data. (2) Divide the data into $r$ stochastically equivalent subgroups. The $r$ subgroups will be of size s. (3) Each subgroup is omitted from the data in turn. This results in $r$ partial or decremented estimates, $\hat{\theta}_{-j}$, each based on the remaining $r-1$ subgroups. (4) From each decremented estimate, calculate a pseudovalue:

$$
\hat{\theta}_{*_{j}}=r \hat{\theta}-(r-1) \hat{\theta}_{-j} .
$$

(5) The jackknife estimate is the mean of the pseudovalues:

$$
\hat{\theta}_{*}=\frac{1}{r} \sum_{j=1}^{r} \hat{\theta}_{*_{j}} .
$$

(6) The variance is calculated from the pseudovalues as if they were independent observations:

$$
\hat{s}_{\theta}^{2}=\frac{\sum_{j=1}^{r}\left(\theta_{*_{j}}-\hat{\theta}_{*}\right)^{2}}{r-1} .
$$

The standard error of estimate for $\theta$ is then

$$
S E_{\hat{\theta}_{*}}=\frac{\hat{s}_{\theta_{*}}}{\sqrt{r}} .
$$

(7) Where many parameters are estimated, the procedure will result in a new data matrix with $r$ rows and $k$ columns, where $r$ is the number of subgroups and $k$ is the number of estimated parameters. The mean of these estimates gives the jackknife parameter estimate, and its variance yields the sampling variance of the mean.

\section{Adaptation of the Jackknife to Factor Rotation}

Normalizing transformations. Normalizing transformations should be used on $\hat{\theta}, \hat{\theta}_{-j}, \hat{\theta}_{* j}$, or $\hat{\theta}_{*}$ in appropriate circumstances. The transformations generally prevent a parameter estimate from falling outside of the allowable range. For example, Clarkson (1979) used no transformations of the factor loadings during the jackknifing process. He reported that many of the jackknifed factor loadings fell outside the allowable range, and he discarded these from subsequent calculations.

In order to prevent such difficulties, the jackknife statistical tests used with the intermax rotation transform the unsquared, rotated factor loadings by the Fisher $z^{\prime}$ transformation (see Winer, 1971, pp. 397-402):

$$
\hat{\phi}=.5 \ln \frac{1+\hat{\theta}}{1-\hat{\theta}},
$$

where $\theta$ is the sample factor loading.

For purposes of jackknife estimation, the squared factor loadings and the intermax criterion function are transformed by the arcsine transformation:

$$
\hat{\phi}=\operatorname{arcsine}(\sqrt{\theta}),
$$

where $\theta$ is the sample squared factor loading or the intermax criterion from Equation 1.

The alignment problem. The rotated factors for the subgroups must somehow be matched (aligned) with appropriate factors from the total sample solution (Clarkson, 1979). The total sample rotated solution is taken as the target matrix. Both the total sample and decremented subsample matrices are transformed by the Fisher $z$ ' transformation. The Euclidian distances are calculated between each pair of total and subsample factors. Subsample factors are aligned with total sample factors by an assignment algorithm (Levin \& Kirkpatrick, 1978, pp. 379-386), so that the distances between the aligned subsample and total sample factors are minimized. Then the jackknife calculations to estimate the loadings and their sampling variances commence.

\section{Jackknife Statistical Tests}

The number of factors. The jackknife procedure results in $r$ (number of subgroups) estimates of INT for each number of factors. The same subgroups are used no matter how many factors are considered. Therefore, a matched sample $t$ test (Kazmier, 1976) can be done to determine whether each successive factor added to the rotated solution improves INT. The null hypothesis is that the new factor produces no gain in INT. The user may specify an alpha value for this test, or by default the program will assume that alpha is 0.05 .

The analysis of variance. The two-way factorial effects are computed, as explained in Winer (1971, pp. 431442). However, the scores used in the cells of the design are the arcsine transformations of the square roots of the squared loadings, and the mean square error variance is estimated as the average of the within-cell error variances. The program assumes that the number of cases per cell is constant and equal to the total number of subgroups. The null probability of each effect is printed.

Significance of individual factor loadings. The point estimates and sampling variances of the individual rotated 
factor loadings are found from their jackknifed Fisher $z^{\prime}$ transformations. A one-tailed $t$ test is applied to each factor loading to test the null hypothesis that it is less than or equal to zero. The program prints the probability of observing a loading given that the null hypothesis is true in the population. The program assumes that the number of cases per cell is constant and equal to the total number of subgroups. Due to computational requirements, 10 or fewer subgroups should be specified for the jackknife procedures (see Clarkson, 1979). Missing data are handled appropriately within each subgroup.

\section{Parametric Estimation Methods}

These estimates assume that the data are multivariate normal and that none of the previously described departures from well-conditioned data are present. Such parameter estimates have advantages: (1) they tend to be relatively less cumbersome computationally, and (2) they may be applied where the raw data are unavailable. As mentioned earlier, the program uses these methods if the input data consist of a factor matrix or a correlation matrix.

The number of factors. The dimensionality is estimated as that which maximizes the intermax criterion.

The analysis of variance. The two-way factorial effects are computed (see Winer, 1971, pp. 431-442), and the mean square error is estimated as the mean of the within-cell variances. The error variances of estimate of each factor loading are computed as follows: The sampling variances of the unrotated principal component loadings are estimated as suggested in Cattell (1978, p. 480). The standard error of estimate for an unrotated principal component factor loading, $\theta$, is estimated as

$$
S E_{\theta^{*}}=\left(\frac{1-\theta^{2}}{N-2}\right)^{1 / 2}\left(\frac{N}{N+1-F}\right)^{1 / 2},
$$

where all symbols are as defined earlier, except that $F$ is the order of the unrotated principal component (e.g., the first, second, third, and so forth). This formula implies that before rotation, the sampling variances of the loadings on the earlier components will tend to be smaller than those on later-extracted components.

When the factor loadings are rotated, the standard errors of the rotated factor loadings can be estimated since the new factor loadings are composites of the principal component loadings for each variable multiplied by weights given in the transformation matrix (Ghiselli, 1964, pp. 302-303; Harman, 1967, pp. 249-272). Given the assumption of stochastic independence of the factor loading estimates, then the error variance of the rotated loadings can be calculated by the formula for the variance of a composite of uncorrelated components (Ghiselli, 1964, pp. 302-303):

$$
S E_{B}^{2}=\sum_{j=1}^{k} S E_{\theta^{*}}^{2} T_{j}^{2},
$$

where subscript $B$ refers to the standard error of the rotated factor loading and $T$ refers to the appropriate ele- ments of the rotation matrix. It is likely that the sampling errors of each factor loading tend to be normally distributed since each rotated factor loading is a weighted composite of the corresponding unrotated factor loadings. Equation 9 gives an estimate of the standard error of each rotated loading, and hence the significance of each loading can be found. The one-tailed $t$ test is used, with degrees of freedom equal to $N-M-1$ for each loading, where $M$ is the number of factors in the solution (Cattell, 1978 , pp. 480-481). When the loadings are squared, then the resultant error distribution for any one factor loading will tend to be similar to a chi-square distribution with one degree of freedom (Myers \& Enrick, 1970). The standard errors of the individual squared factor loadings are estimated by:

$$
S E_{B^{2}}=1.4142 S E_{B} .
$$

The pooled standard errors of the individual rotated and squared loadings provide the estimate of the mean square error for the analysis of variance of the rotated factor matrix.

Significance of factor loadings. Equation 9 gives the standard error of estimate for each rotated factor loading. The one-tailed $t$ test is used to test whether each factor loading is significantly greater than zero.

\section{COMPUTATIONAL EXAMPLE}

Harman's (1967; pp. 13-14, 137, 161-163, 203-204, 229-230, 309-310, 340, 349) analysis example of five socioeconomic factors was chosen to illustrate the intermax rotation method and its associated significance tests.

\section{RESULTS}

\section{The Jackknife Estimates}

Number of factors. The intermax criterion was calculated for the full sample and for each of the 12 subsamples for one to five factors. The paired sample $t$ test (with alpha of 0.05 ) was computed as each additional factor was added to the solution to determine dimensionality. The $t$ test on the intermax criterion, INT, indicated that the second factor was the last one which produced a significant improvement in simple structure. The jackknifed intermax criterion values, INT, for factors one through three were respectively 0.00 (by definition), 0.9990 , and 0.9869 . The $t$ tests were, respectively, $59.15(p<$ $.0005)$, and $-1.80(p>.50)$ for gains in INT from adding factors two and three. Hence, the two-factor solution was accepted. The principal component, varimax, intermax, and jackknifed intermax solutions are given in Table 2, along with the significance of each rotated factor loading in the intermax solutions.

Analysis of variance. The analyses of variance of the squared factor loadings are given in Table 3 . The variables are not significantly different in communalities, and the rotated factors are not significantly different in magnitude. The interaction effect is significant $[F=34.66$, $p<.01]$ and large (jackknifed INT $=0.9990$ ). 
Table 2

The Principal Components, Varimax, and Intermax Solutions for Five Socioeconomic Variables

\begin{tabular}{|c|c|c|c|c|c|c|c|c|}
\hline \multirow[b]{2}{*}{ Variable } & \multicolumn{2}{|c|}{ Principal Components } & \multicolumn{2}{|c|}{ Varimax } & \multicolumn{2}{|c|}{ Intermax } & \multicolumn{2}{|c|}{ Jackknifed Intermax } \\
\hline & $\mathbf{I}_{\dagger}^{\dagger}$ & II†† & I\$ & II** & I§ & II** & I§ & II** \\
\hline Total Population & 0.5810 & 0.8064 & 0.0160 & 0.9938 & -0.1529 & $0.9820^{*}$ & 0.0210 & $0.9258 *$ \\
\hline Median School & 0.7671 & -0.5448 & 0.9408 & -0.0088 & $0.9285^{*}$ & 0.1510 & $0.8237^{*}$ & -0.0046 \\
\hline Employment & 0.6724 & 0.7260 & 0.1370 & 0.9801 & -0.0314 & $0.9890^{*}$ & 0.0883 & $0.9894 *$ \\
\hline Professionals & 0.9324 & -0.1043 & 0.8248 & 0.4471 & $0.7369 *$ & $0.5806^{*}$ & $0.6053^{*}$ & $0.3091 *$ \\
\hline House Value & 0.7911 & -0.5582 & 0.9682 & -0.0060 & $0.9552 *$ & 0.1584 & $0.8675^{*}$ & 0.0068 \\
\hline
\end{tabular}

Note-The variables are explained more fully in Harman (1967). ${ }^{*} p<.05$ by the one-tailed $t$ test for null hypothesis of zero or smaller loading. $\uparrow \dagger \dagger$ These are the loadings on the first two principal components. $\S * *$ These are various rotated solutions, as labeled.

Significance of factor loadings. The jackknifed rotated factor loadings are shown in Table 2. Factor loadings of 0.31 or more were all significantly greater than zero by the $t$ test (alpha $=0.05$ ).

\section{The Parametric Estimates}

Number of factors. The intermax criteria for one, two, and three factors respectively were 0.00 (by definition), 0.9970 , and 0.9833 . Since the intermax criterion declined when the number of factors was increased to three, the two-factor solution was accepted.

Analysis of variance. The variables did not differ in communality, and the rotated factors did not differ in magnitude. The interaction effect was significant $[F=3.82$, $p<.05$ ], and large (INT $=0.9970$ ).

Significance of individual factor loadings. All rotated loadings of 0.58 or greater were significantly greater than zero by the one-tailed $t$ test at the .05 level.

\section{CONCLUSIONS}

\section{The Intermax Rotation Method}

The rationale for the intermax rotation method is simpler than that of the varimax method. The normalized varimax method's results appear similar to those of intermax because the varimax normalization process tends to remove the variables effect from the factor matrix. Further study of analytical methods may simplify the calculation of the intermax rotation.

\section{The Intermax Significance Tests}

The intermax rotation method leads to new significance

Table 3

The Analyses of Variance of the Intermax Solutions

\begin{tabular}{lccccc}
\hline \multicolumn{7}{c}{ Source } & $\begin{array}{l}\text { Sum of } \\
\text { Squares }\end{array}$ & $d f$ & Squares & $F$ & $p$ \\
\hline \multicolumn{7}{c}{ Jackknife Analysis of Variance Summary Table } \\
Factors $(F)$ & 0.7690 & 1 & 0.7690 & 1.63 & $>.05$ \\
Variables $*(V)$ & 0.5212 & 4 & 0.1303 & $<1$ & $>.05$ \\
$F \times V$ & 65.4434 & 4 & 16.3609 & 34.66 & $<.05$ \\
Error & 51.9310 & 110 & 0.4721 & & \\
\multicolumn{7}{c}{ Parametric Analysis of Variance Summary } \\
Factors $(F)$ & 0.0000 & 1 & 0.0000 & $<1$ & $>.05$ \\
Variables * $(V)$ & 0.0013 & 4 & 0.0013 & $<1$ & $>.05$ \\
$F \times V$ & 1.6919 & 4 & 0.4230 & 3.82 & $<.05$ \\
Error & 12.1880 & 110 & 0.1108 & & \\
*The variables are explained more fully in Harman & $(1967)$.
\end{tabular}

*The variables are explained more fully in Harman (1967). tests for the number of factors, differences in rotated factor magnitudes, differences in communalities, and the existence of simple structure or factorial invariance. In addition, either jackknife or parametric methods can be used to test the significance of a rotated factor loading. These statistical tests focus on achieving simple structure. They are different from, but complementary to, those which previously existed.

The jackknife method of estimating the parameters and the sampling variances is preferred since it makes fewer assumptions about the nature of the data. The inferential tests can also be conducted where only a factor matrix or correlation matrix is available. In that case, more restrictive assumptions are made about the nature of the raw data.

The jackknife methods can be elaborated. For example, dimensionality might be estimated by redefining INT to account for sampling error in the rotated loadings, or to be similar to omega square. Paired $t$ tests could detect on which of two factors a variable loaded most highly. Various analysis of variance comparisons could be made on the factor matrix. The performance of different methods of dimension analysis (e.g., maximum likelihood factor analysis and nonmetric dimension analysis) could be compared.

\section{The Example Problem}

The example problem was chosen to illustrate the intermax methods. The number of factors, the significant interaction effect, and the significance of each factor loading were found by both the jackknife and the parametric methods. The rotated factor loadings estimated by the jackknife method may tend to be more accurate than those from the parametric method. Monte Carlo studies of samples from a known population could provide direct evidence of the relative accuracy of these methods. Future applications of the intermax rotation method and its associated hypothesis tests to other and larger data sets will provide better evidence of its general usefulness and validity.

\section{AVAILABILITY}

The user's guide and program source listing will be provided free of charge to interested readers. 


\section{REFERENCES}

Balloun, J. L, \& KeARNS, J. (1975). An approach to an orthogonal simple structure solution by maximizing test-factor interaction effects among squared factor loadings. British Journal of Mathematical \& Statistical Psychology, 28, 63-70.

Balloun, J. L., \& OUMLIL, A. B. (1986). JACKKNIFE: A generalpurpose package for generating multivariate jackknife analyses. $B e$ havior Research Methods, Instruments, \& Computers, 18, 47-49.

BisSELl, A. F. (1977). The jackknife. Bulletin in Applied Statistics, 4, 55-64.

CATTELL, R. B. (1966). Handbook of multivariate experimental psychology. Chicago: Rand-McNally.

CATTELL, R. B. (1978). The scientific use of factor analysis in the behavioral and life sciences. New York: Plenum Press.

Clarkson, D. B. (1979). Estimating the standard errors of rotated factor loadings by jackknifing. Psychometrika, 44, 297-314.

GHISELlI, E. E. (1964). Theory of psychological measurement. New York: McGraw-Hill.

Hair, J. F., Anderson, R. E., Tatham, R. L., \& Grablowsky, B. J. (1979). Multivariate data analysis. Tulsa, OK: Petroleum Publishing.

Harman, H. H. (1967). Modern factor analysis (2nd ed.). Chicago: University of Chicago Press.

KAZMIER, L. J. (1976). Schaum's outline of theory and problems of business statistics. New York: McGraw-Hill.

KIM, J. , \& MUELLER, C. W. (1978). Factor analysis: Statistical methods and practical issues. Sage University Paper Series on Quantitative Applications in the Social Sciences (No. 07-014). Beverly Hills: Sage Publications.

LeVIN, R. I., \& KiRKPATRICK, C. A. (1978). Quantitative approaches to management (4th ed.). New York: McGraw-Hill.

Myers, B. L., \& ENRICK, N. L. (1970). Statistical functions. Kent, OH: Kent State University Press.

Miller, R. G. (1974). The jackknife-a review. Biometrika, 61, 1-17.

Mosteller, F., \& TUKEY, J. W. (1968). Data analysis including statistics. In G. Lindzey \& E. Aronson (Eds.), The handbook of social psychology (Vol. 2). Reading, MA: Addison-Wesley.

Nie, N. H., Hull, C. H., Jenkins, J. G., Steinbrenner, K., \& Bent, D. H. (1975). Statistical package for the social sciences (2nd ed.). New York: McGraw-Hill.

Oumlil, A. B. (1983). Economic change and consumer shopping behavior. New York: Praeger.

Thurstone, L. L. (1947). Multiple factor analysis. Chicago: University of Chicago Press.

TUKEY, J. W. (1980). Methodological comments focused on opportunities. In P. R. Monge \& J. N. Capella (Eds.), Multivariate techniques in human communication research. New York: Academic Press.

WINER, B. J. (1971). Statistical principles in experimental design (2nd ed.). New York: McGraw-Hill.

(Revision accepted for publication March 7, 1986.) 